\title{
An electrostatic model for zeros of classical Laguerre polynomials perturbed by a rational factor
}

\author{
Luis Alejandro Molano Molano
}

Received: 15 January 2014/ Accepted: 23 April 2014/Published online: 5 June 2014

(C) The Author(s) 2014. This article is published with open access at Springerlink.com

\begin{abstract}
In this paper, we study an electrostatic model for zeros of polynomials orthogonal with respect to inner product

$\langle p, q\rangle_{\alpha, \eta}=\int_{0}^{\infty} p(x) q(x) \frac{1}{x-\eta} \mathrm{d} \mu(x)$,

where $\mathrm{d} \mu(x)$ is the classical Laguerre measure $\mathrm{e}^{-x} x^{\alpha} \mathrm{d} x$, with $\alpha>-1$ and $\eta<0$.
\end{abstract}

Keywords Orthogonal polynomials - Zeros ·

Electrostatic model

\section{Introduction}

In pioneering works of Heine and Stieltjes, (see [4] and [6]), a nice electrostatic interpretation of the zeros of the classical sequences of orthogonal polynomials was established. To illustrate these ideas, consider the two-parameter sequence of Jacobi polynomials $\left\{P_{n}^{\alpha, \beta}(x)\right\}_{n \in \mathbb{N}}$, orthogonal with respect to the weight $\omega(x)=(1-x)^{\alpha}(1+x)^{\beta}$, (where $\alpha, \beta>-1)$, supported on $[-1,1]$. It is well known that the zeros of $P_{n}^{\alpha, \beta}(x)$ are real, simple and these lie into the interval of orthogonality $[-1,1]$. Given $n \in \mathbb{N}$, if two charges of strength $\frac{\beta+1}{2}$ and $\frac{\alpha+1}{2}$ are put at -1 and 1

L. A. M. Molano is equally contributed to this work.

L. A. M. Molano ( $\square)$

Facultad Seccional Duitama, Licenciatura en matemáticas y estadística, Universidad Pedagógica y Tecnológica de Colombia, Duitama, Colombia

e-mail: luis.molano01@uptc.edu.co respectively, $n$ movable unit charges are distributed freely in $(-1,1)$, and assuming that the interaction obeys the logarithmic potential law, then there exists exactly one equilibrium position, the global minimum of total energy. Such equilibrium is reached precisely in the vector of zeros associated with $P_{n}^{\alpha, \beta}(x)$. This interpretation can also be made in terms of the family of Laguerre polynomials $\left\{L_{n}^{\alpha}(x)\right\}_{n \in \mathbb{N}}$ with $\omega(x)=\mathrm{e}^{-x} x^{\alpha}$ as weight of orthogonality, where $\alpha>-1$ and supported on $[0, \infty)$. It is enough fix one charge with strength $\frac{\alpha+1}{2}$ at the origin, and with the additional condition that the mean arithmetic of the positions of the $n$ movable charges is uniformly bounded. So the unique global minimum of total energy coincides with the vector of zeros of the rescaled polynomial $L_{n}^{\alpha}\left(\frac{n+\alpha}{C} x\right)$, where $C$ is the constant of uniform boundedness. The cornerstone that supports such results is the second-order linear differential equation

$\sigma(x) p^{\prime \prime}(x)+\tau(x) p^{\prime}(x)+\lambda_{n} p(x)=0$,

(where $\sigma(x)$ and $\tau(x)$ are polynomials with ${ }^{\circ}(\sigma(x)) \leq 2$ and $\left.{ }^{\circ}(\tau(x))=1\right)$ that satisfy the families of classical polynomials. In [5], an electrostatic model for zeros of polynomials orthogonal with respect to weights supported on intervals bounded or unbounded is presented, which generalizes the work of Heine and Stieltjes. There, the total energy contains external fields which are closely related to the respective weight function, and in this way, without to limit the arithmetic mean or rescale the variable, the zeros of $L_{n}^{\alpha}(x)$ still provide the unique equilibrium position, if a component given for the external field $\phi(x)=\frac{x}{2}$ is added to the total energy. Inspired by the above ideas, in this paper, we propose an electrostatic model for zeros of the monic polynomials $\left\{G_{n}^{\alpha}(x)\right\}_{n \in \mathbb{N}}$, orthogonal with respect to inner product 


$$
\langle p, q\rangle_{\alpha, \eta}=\int_{0}^{\infty} p(x) q(x) \frac{\mathrm{e}^{-x} x^{\alpha}}{x-\eta} \mathrm{d} x,
$$

where $\eta<0$. This is, we work with the classical Laguerre weight perturbed by a rational factor. Such perturbations are known in the literature as canonical Geronimus transformations, and a study of this kind of polynomials can be seen in [2], where is obtained relative asymptotics of these polynomial as well as Mehler-Heine type formula and a Plancherel-Rotach type formula for the rescaled polynomials. In this way, the structure of this manuscript is as follows. In "Preliminaries", we present some auxiliary results about the sequences $\left\{L_{n}^{\alpha}(x)\right\}_{n \in \mathbb{N}}$ and $\left\{G_{n}^{\alpha}(x)\right\}_{n \in \mathbb{N}}$. In "Holonomic equation", we deduce using standard techniques, a second-order linear differential equation satisfied for every $G_{n}^{\alpha}(x)$ and in "An electrostatic model", we discuss a electrostatic interpretation of the zeros of these polynomials.

\section{Preliminaries}

Let $\left\{L_{n}^{\alpha}(x)\right\}_{n \in \mathbb{N}}$ be the sequence of classical Laguerre monic polynomials, orthogonal with respect to the inner product

$$
\langle p, q\rangle_{\alpha}=\int_{0}^{\infty} p(x) q(x) \mathrm{d} \mu_{\alpha}
$$

where $\mathrm{d} \mu=\mathrm{e}^{-x} x^{\alpha} \mathrm{d} x$, with $\alpha>-1$. In the next proposition, some properties of classical Laguerre polynomials which we will use in the sequel are summarized (see [1] or [7]).

Proposition 1 For every $n \in \mathbb{N}$, the polynomials $\left\{L_{n}^{\alpha}(x)\right\}_{n \in \mathbb{N}}$ hold

1. (three-term recurrence relation).

$x L_{n}^{\alpha}(x)=L_{n+1}^{\alpha}(x)+(2 n+1+\alpha) L_{n}^{\alpha}(x)+n(n+\alpha) L_{n-1}^{\alpha}(x)$.

with $L_{0}^{\alpha}(x)=1$ and $L_{1}^{\alpha}(x)=x-(\alpha+1)$.

2. (Structure relation)

$$
L_{n}^{\alpha}(x)=L_{n}^{\alpha+1}(x)+n L_{n-1}^{\alpha+1}(x) .
$$

3.

$$
x\left(L_{n}^{\alpha}(x)\right)^{\prime}=n L_{n}^{\alpha}(x)+n(n+\alpha) L_{n-1}^{\alpha}(x) .
$$

4. $L_{n}^{\alpha}(x)$ satisfies the differential equation

$$
x y^{\prime \prime}+(\alpha+1-x) y^{\prime}=-n y \text {. }
$$

In the other hand, let $\left\{G_{n}^{\alpha}(x)\right\}_{n \in \mathbb{N}}$ be the sequence of monic polynomials, orthogonal with respect to inner product

$$
\langle p, q\rangle_{\alpha, \eta}=\int_{0}^{\infty} p(x) q(x) \frac{\mathrm{d} \mu_{\alpha}}{(x-\eta)}, \quad \eta<0 .
$$

To give an efficient algebraic relation between families $\left\{G_{n}^{\alpha}(x)\right\}_{n \in \mathbb{N}}$ and $\left\{L_{n}^{\alpha}(x)\right\}_{n \in \mathbb{N}}$, in [2] the connection formula has been found

$$
G_{n}^{\alpha}(x)=L_{n}^{\alpha}(x)+\varsigma_{n} L_{n-1}^{\alpha}(x),
$$

with

$\varsigma_{n}=n+\beta_{n}$.

The constant $\beta_{n}$ has the explicit representation

$\beta_{n}=\frac{\eta}{n} \frac{I(\alpha-1, n)}{I(\alpha, n-1)}$,

where

$$
I(\alpha, n)=\int_{0}^{\infty} \frac{x^{n}}{(x-\eta)^{n+1}} \mathrm{~d} \mu_{\alpha},
$$

and is clear that $I(\alpha, n)>0$, and so $\beta_{n}<0$. Even more, it is known his asymptotic behaviour, namely

$$
\beta_{n} \approx-\frac{\sqrt{-\eta}}{n^{3 / 2}} \text {. }
$$

\section{Holonomic equation}

Using standard techniques, we will deduct a second-order linear differential equation that $G_{n}^{\alpha}(x)$ satisfy. In effect, taking derivatives in (5), multiplying by the factors $(\alpha+$ $1-x)$ and $x$, respectively, and by adding the resulting equations, we have

$$
\begin{aligned}
& x\left(G_{n}^{\alpha}\right)^{\prime \prime}(x)+(\alpha+1-x)\left(G_{n}^{\alpha}\right)^{\prime}(x) \\
& =\left[x\left(L_{n}^{\alpha}\right)^{\prime \prime}(x)+(\alpha+1-x)\left(L_{n}^{\alpha}\right)^{\prime}(x)\right] \\
& \quad+\varsigma_{n}\left[x\left(L_{n-1}^{\alpha}\right)^{\prime \prime}(x)+(\alpha+1-x)\left(L_{n-1}^{\alpha}\right)^{\prime}(x)\right],
\end{aligned}
$$

and using (4) we get

$$
\begin{aligned}
& x\left(G_{n}^{\alpha}\right)^{\prime \prime}(x)+(\alpha+1-x)\left(G_{n}^{\alpha}\right)^{\prime}(x) \\
& \quad=-n\left[L_{n}^{\alpha}(x)+\varsigma_{n} L_{n-1}^{\alpha}(x)\right]+\varsigma_{n} L_{n-1}^{\alpha}(x) .
\end{aligned}
$$

And considering (5), we find the equation

$$
x\left(G_{n}^{\alpha}\right)^{\prime \prime}(x)+(\alpha+1-x)\left(G_{n}^{\alpha}\right)^{\prime}(x)+n G_{n}^{\alpha}(x)=\varsigma_{n} L_{n-1}^{\alpha}(x) .
$$

On the other hand, taking derivatives in (5) and multiplying by $x$, we have 
$x\left(G_{n}^{\alpha}\right)^{\prime}(x)=x\left(L_{n}^{\alpha}\right)^{\prime}(x)+\varsigma_{n} x\left(L_{n-1}^{\alpha}\right)^{\prime}(x)$

and using (2) and (1), we obtain respectively

$$
\begin{aligned}
x\left(G_{n}^{\alpha}\right)^{\prime}(x)= & n L_{n}^{\alpha}(x)+\left[n(n+\alpha)+(n-1) \varsigma_{n}\right] \\
& \times \mathrm{L}_{\mathrm{n}-1}^{\alpha}(\mathrm{x})+\varsigma_{\mathrm{n}}(\mathrm{n}-1)(\mathrm{n}-1+\alpha) \mathrm{L}_{\mathrm{n}-2}^{\alpha}(\mathrm{x}),
\end{aligned}
$$

and

$$
\begin{aligned}
x\left(G_{n}^{\alpha}\right)^{\prime}(x)= & n L_{n}^{\alpha}(x)+\left[n(n+\alpha)+(n-1) \varsigma_{n}\right] L_{n-1}^{\alpha}(x) \\
& +\varsigma_{n}\left[x L_{n-1}^{\alpha}(x)-L_{n}^{\alpha}(x)-(2 n-1+\alpha) L_{n-1}^{\alpha}(x)\right] \\
= & {\left[n-\varsigma_{n}\right] L_{n}^{\alpha}(x)+\left[n(n+\alpha)+(n-1) \varsigma_{n}\right.} \\
& \left.+x \varsigma_{n}-\varsigma_{n}(2 n-1+\alpha)\right] L_{n-1}^{\alpha}(x),
\end{aligned}
$$

and as a consequence

$x\left(G_{n}^{\alpha}\right)^{\prime}(x)=-\beta_{n} L_{n}^{\alpha}(x)+\left[\varsigma_{n} x-\beta_{n}(n+\alpha)\right] L_{n-1}^{\alpha}(x)$.

Then, from (5) and (8), we have a system of two equations with unknowns $L_{n}^{\alpha}(x)$ and $L_{n-1}^{\alpha}(x)$, namely

$$
\left\{\begin{array}{l}
L_{n}^{\alpha}(x)+\varsigma_{n} L_{n-1}^{\alpha}(x)=G_{n}^{\alpha}(x) \\
-\beta_{n} L_{n}^{\alpha}(x)+\left[\varsigma_{n} x-\beta_{n}(n+\alpha)\right] L_{n-1}^{\alpha}(x)=x\left(G_{n}^{\alpha}\right)^{\prime}(x),
\end{array}\right.
$$

then if

$$
\begin{aligned}
\Delta(x, n, \alpha) & =\operatorname{det}\left(\begin{array}{cc}
1 & \varsigma_{n} \\
-\beta_{n} & \left(\varsigma_{n} x-\beta_{n}(n+\alpha)\right)
\end{array}\right) \\
& =x \varsigma_{n}-\beta_{n}\left(\alpha-\beta_{n}\right),
\end{aligned}
$$

clearly

$$
\begin{aligned}
L_{n-1}^{\alpha}(x) & =\frac{\operatorname{det}\left(\begin{array}{cc}
1 & G_{n}^{\alpha}(x) \\
-\beta_{n} & x\left(G_{n}^{\alpha}\right)^{\prime}(x)
\end{array}\right)}{\Delta(x, n, \alpha)} \\
= & \frac{x\left(G_{n}^{\alpha}\right)^{\prime}(x)+\beta_{n} G_{n}^{\alpha}(x)}{\Delta(x, n, \alpha)} .
\end{aligned}
$$

and substituting in (7) we get

$$
\begin{gathered}
x\left(G_{n}^{\alpha}\right)^{\prime \prime}(x)+(\alpha+1-x)\left(G_{n}^{\alpha}\right)^{\prime}(x)+n G_{n}^{\alpha}(x) \\
=\frac{\varsigma_{n} x\left(G_{n}^{\alpha}\right)^{\prime}(x)}{\Delta(x, n, \alpha)}+\frac{\varsigma_{n} \beta_{n} G_{n}^{\alpha}(x)}{\Delta(x, n, \alpha)}
\end{gathered}
$$

or equivalently

$$
\begin{aligned}
& x\left(G_{n}^{\alpha}\right)^{\prime \prime}(x)+\left[(\alpha+1-x)-\frac{\varsigma_{n} x}{\Delta(x, n, \alpha)}\right]\left(G_{n}^{\alpha}\right)^{\prime}(x) \\
& +\left[n-\frac{\varsigma_{n} \beta_{n}}{\Delta(x, n, \alpha)}\right] G_{n}^{\alpha}(x)=0 .
\end{aligned}
$$

We summarize the results in the next

Proposition 2 The $\mathrm{n}$-th monic polynomial $G_{n}^{\alpha}(x)$ is a solution of the second-order linear differential equation $x \phi^{\prime \prime}(x)+B(x, n) \phi^{\prime}(x)+C(x, n) \phi(x)=0$,

where

$$
B(x, n)=(\alpha+1-x)-\frac{x}{x-\omega_{n}},
$$

and

$$
C(x, n)=n-\frac{\beta_{n}}{x-\omega_{n}}
$$

with

$\omega_{n}=\frac{\beta_{n}}{\varsigma_{n}}\left(\alpha-\beta_{n}\right)$

Remark 1 Note that $\omega_{n}$ can be expressed as $\omega_{n}=\frac{1}{n} \frac{\beta_{n}}{1+\frac{\beta_{n}}{n}}\left(\alpha-\beta_{n}\right)$,

and taking into account (6) is clear that if $n \rightarrow \infty$ then $\beta_{n} \rightarrow 0$ and $\frac{\beta_{n}}{n} \rightarrow 0$, as a consequence $\omega_{n} \rightarrow 0$.

\section{An electrostatic model}

Now, we give an electrostatic interpretation for the zeros of $G_{n}^{\alpha}(x)$. Let $\left\{\rho_{n, k}\right\}_{k=1}^{n}$ be their zeros arranged in a increasing order. Given that such zeros are real and simples, it is easy check that for $k=1, \ldots, n$

$\frac{\left(G_{n}^{\alpha}\right)^{\prime \prime}\left(\rho_{n, k}\right)}{2\left(G_{n}^{\alpha}\right)^{\prime}\left(\rho_{n, k}\right)}=\sum_{j=1, j \neq k}^{n} \frac{1}{\rho_{n, k}-\rho_{n, j}}$.

Now we evaluate (10) in $\rho_{n, k}$ for to obtain

$$
\rho_{n, k}\left(G_{n}^{\alpha}\right)^{\prime \prime}\left(\rho_{n, k}\right)+B\left(\rho_{n, k}, n\right)\left(G_{n}^{\alpha}\right)^{\prime}\left(\rho_{n, k}\right)=0,
$$

or equivalently

$$
\frac{\left(G_{n}^{\alpha}\right)^{\prime \prime}\left(\rho_{n, k}\right)}{2\left(G_{n}^{\alpha}\right)^{\prime}\left(\rho_{n, k}\right)}=-\frac{B\left(\rho_{n, k}, n\right)}{2 \rho_{n, k}},
$$

and using (11) we get

$$
\begin{aligned}
\frac{B(x, n)}{2 x} & =\frac{(\alpha+1-x)}{2 x}-\frac{1}{2\left(x-\omega_{n}\right)} \\
& =\frac{(\alpha+1)}{2 x}-\frac{1}{2}-\frac{1}{2\left(x-\omega_{n}\right)},
\end{aligned}
$$

so, from (12) we have

$$
\sum_{j=1, j \neq k}^{n} \frac{1}{\rho_{n, k}-\rho_{n, j}}=-\frac{(\alpha+1) / 2}{\rho_{n, k}}+\frac{1}{2}+\frac{1 / 2}{\left(\rho_{n, k}-\omega_{n}\right)} .
$$

To describe an electrostatic model, consider a fixed positive charge of strength $(\alpha+1) / 2$ at the origin, a fixed 
negative charge of strength $-1 / 2$ at $\omega_{n}$, and $n$ movable unit charges distributed in $(0, \infty)$, and suppose that such unit charges are in presence of an external field $\varphi(x)=x / 2$. We will suppose that the charges interact with each other through a logarithmic potential under an external field. So the total energy $E(X)$ of the system (with $X=\left(x_{1}, \ldots, x_{n}\right)$ ) is given by the sum of the following components: the mutual interaction of these charges, the external fields created by fixed charges and moreover the external field $\varphi(x)$. Mathematically this is

$$
\begin{aligned}
E(X)= & -\sum_{1 \leq k<j \leq n} \ln \left|x_{k}-x_{j}\right|-\frac{(\alpha+1)}{2} \sum_{j=1}^{n} \ln \left|x_{j}\right| \\
& +\frac{1}{2} \sum_{j=1}^{n}\left(\ln \left|x_{j}-\omega_{n}\right|-x_{j}\right),
\end{aligned}
$$

and it is known that the electrostatic equilibrium of the system, that is, a configuration of positions $X^{*}$ where the $n$ movable unit charges are in equilibrium, occurs in stationary points of the total energy $E(X)$. We will prove that effectively, $X^{*}=\left(\rho_{n, 1}, \rho_{n, 2}, \ldots, \rho_{n, n}\right)$ provides a critical point for the total energy $E(X)$. Indeed, form (14) and for $k=1, \ldots, n$

$$
\frac{\partial E}{\partial x_{i}}(X)=-\sum_{j \neq i} \frac{1}{x_{i}-x_{j}}-\frac{(\alpha+1)}{2 x_{i}}+\frac{1}{2\left(x_{i}-\omega_{n}\right)}+\frac{1}{2} .
$$

But from (13) $\frac{\partial E}{\partial x_{i}}\left(X^{*}\right)=0$, which completes the proof.

Remark 2 Note that the external field $\varphi(x)=x / 2$ is the same that in [5] is considered for to model the electrostatic behaviour of zeros of classical Laguerre polynomials. Furthermore, note that in our model, there is a fixed charge that depends of $n$ and his strength is negative. In this way, in [3], an electrostatic model with such features for JacobiKoornwinder orthogonal polynomials is obtained.

Conflict of interest The author declare that he has no competing interests.

Open Access This article is distributed under the terms of the Creative Commons Attribution License which permits any use, distribution, and reproduction in any medium, provided the original author(s) and the source are credited.

\section{References}

1. Chihara, T.S.: An introduction to orthogonal polynomials. Mathematics and its Applications 13. Gordon and Breach, New York (1978)

2. Fejzullahu, B.X.: Asymptotics for orthogonal polynomials with respect to the Laguerre measure modified by a rational factor. Acta Sci. Math. Szeged. 77, 73-85 (2011)

3. Grünbaum, F.A.: Variations on a theme of Heine and Stieltjes: an electrostatic interpretation of the zeros of certain polynomials. J. Comput. Appl. Math. 99, 189-194 (1998)

4. Heine, E.: Handbuch der Kugelfunktionen, vol, 2nd edn. II. G. Reimer, Berlin (1878)

5. Ismail, M.E.H.: An electrostatics model for zeros of general orthogonal polynomials. Pacific J. Math. 193, 355-369 (2000)

6. Stieltjes, T.J.: Sur certain polynômes que vérifient une équation diférentielle linéaire du second ordre et sur la théorie des fonctions de Lamé. Acta Math. 6, 321-326 (1885)

7. Szegö, G.: Orthogonal polynomials. Amer. Math. Soc. Colloq. Publ. 23, Amer. Math. Soc., Providence (1975) 